\title{
Clinical application of electromyography in patients with myofascial pain syndrome: a case report
}

\author{
M. I. Soikher ${ }^{1}$, M. G. Soikher ${ }^{1}$, G. Slavicek ${ }^{2}$ \\ ${ }^{1}$ Institute of Biotechnologies and Interdisciplinary Dentistry, Moscow, Russia \\ ${ }^{2}$ Steinbeis Transfer Institut Biotechnology in Interdisciplinary Dentistry der Steinbeis Hochschule Berlin, Stuttgart, Germany
}

Received November 2, 2009; Accepted January 10, 2010

\begin{abstract}
The aim of this case report is to give comprehensive information on the clinical use of surface electromyography in clinical practice during diagnostics and treatment. A 23-year-old female patient is presented with complaints of fatigue and pain in chewing muscles with deterioration of the symptoms after mastication. In addition, discomfort while clenching, sleeping disorders and headaches are reported by the patient.

Surface electromyography is performed, using the following parameters: bilateral symmetry coefficient for temporalis muscles; bilateral symmetry coefficient for masseter muscles; lateral mandible displacement index; bilateral symmetry coefficient for sternocleidomastoideus muscles; sternocleidomastoideus muscles functional activity coefficient; impact potential of examined muscles. The parameters are used not only in diagnostics, but also in evaluating the treatment progress.

It can be concluded application of EMG in clinical practice is useful in the situations such as screening for functional activity of chewing muscles disorders, development of evidence-based treatment protocols and diagnosis of combined dental and neurological pathology.
\end{abstract}

Keywords: craniomandibular disorder, surface electromyography, EMG, chewing muscles, temporomandibular joint, posture

\section{Introduction}

Automatic devices for medical and biological data collection and processing software can significantly improve diagnostic potential of modern medicine. Electromyography is a technique for evaluating nervous and muscular systems based upon registration of the electric signal of muscles $[1,2]$.

Electromyography was introduced by Vvedensky N.E., who used a telephone device in 1884. The first graphic record of human electromyogram was performed in 1907 by H. Piper. Intense research of electromyography as clinical diagnostic

Correspondence: Dr. Michael I. Soikher, Institute of Biotechnologies and Interdisciplinary Dentistry, 119146 Moscow, Russia.

E-mail: msoiher@yandex.ru tool started in the 1930s and 1940s. Electromyography contributed to the development of a scientific theory on morphological and functional collaboration between nerves and muscles. A deep understanding on the nature of muscle electrical activity in physiological and pathological conditions could be developed.

Unfortunately, dentists pay little attention to the assessment of muscle condition in their routine practice, if they do at all. However, studies report that $80-90 \%$ of all temporomandibular joint dysfunctions are related to impaired coordination of muscles $[3,4]$. Condition of muscles cannot be objectively assessed by clinical examination alone. Bio-instrumental techniques of muscle evaluation are widely used in neuromuscular dentistry and provide objective data on whether muscular activity is adequate or inadequate; furthermore, they support the clinician to monitor the response to the applied treatment and, if indicated, to modify treatment systematically.

Every organism is trying to maintain optimal functions as it grows and develops by using compensatory mechanisms (dentoalveolar, vertical, joint and neuromuscular compensation). Neuromuscular system enables movements of all chewing organ structures and creates functional prototypes of these movements after receiving proprioceptive impulses from other structures and internal control system. Thus, the first line approach in the chewing system function assessment should include standardized protocols for neuromuscular component evaluation. Electromyography is an electrophysiological technique for evaluating neuromuscular system that provides valuable diagnostic information about localization of the jaw and masseter and neck muscle condition.

This article should demonstrate the use of surface EMG of masseter, temporalis and neck muscles at initial visit and during treatment to support the clinician during the treatment of the individual patient.

\section{Material and method}

EMG is performed with a portable electromyography FREELY ${ }^{\circledR}$ (De Gotzen, Milan, Italy) with extended software. Original signal is amplified (capacity to amplify - 150, frequency bandwidth $-0-10 \mathrm{kHz}$, input signal peak to peak value $0-2000 \mathrm{mV}$ ) 
by differential amplifier with high rejection ratio (CMRR of $105 \mathrm{~dB}$ within limits of $0-60 \mathrm{~Hz}$, input impedance $10 \mathrm{G} \Omega$ ). Analogue-to-digital conversion is performed (resolution 12b, sampling rate $2230 \mathrm{~Hz} \mathrm{~A} / \mathrm{D}$ ) and digital data are filtered (highpass filter set at $30 \mathrm{~Hz}$ and low-pass filter set at $400 \mathrm{~Hz}$, bandstop for common $50 \pm 60 \mathrm{~Hz}$ noise). The signals are averaged over $25 \mathrm{~ms}$, with muscle activity assessed as the root mean square (rms) of the amplitude (unit: $\mu \mathrm{V}$ ). EMG signals are recorded for further analysis.

Disposable silver/silver chloride bipolar electrodes with a $10 \mathrm{~mm}$ diameter and $21 \mathrm{~mm}$ inter-electrode distance are used to record EMG from masseter, temporalis anterior and sternocleidomastoideus muscles during testing. One reference electrode is placed on patient's forehead. Impulses from sternocleidomastoideus muscles are recorded to elucidate how these postural muscles contribute to head positioning and collaborate with chewing muscles. Bipolar surface electrodes is positioned on the muscular bellies parallel to muscular fibers as previously described [5-10]: temporalis anterior - vertically along the anterior margin of the muscle; masseter and sternocleidomastoideus - parallel to muscle fibers. Electrodes are applied to alcohol cleaned skin to decrease impedance. Testing is started 5-6 min later, after the application of conductive gel.

\section{Standardization recording (clenching on cotton rolls)}

At first, a standardization recording at maximum voluntary clenching on equal density cotton rolls for 5 seconds is performed. During the recording, patients sit with their head unsupported and are asked to maintain a natural upright position and to clench their teeth as hard as possible in intercuspidal position.

Two 10-mm thick cotton rolls are positioned on the mandibular second premolar/first molars of each patient. Standardization provided reference EMG values for a subsequent normalization. For each of the four analyzed muscles (right and left masseter; right and left temporalis muscle), the mean EMG potential (rms of the amplitude) is set at $100 \%$, and all EMG potentials obtained during MVC directly performed on the occlusal surfaces (see below) are expressed as a percentage of this value (unit: $\mathrm{mV} / \mathrm{mV}$ ).

\section{Maximum voluntary clenching (MVC)}

EMG activity is recorded during a 5-s maximum voluntary clenching test (MVC). The patient is asked to clench as hard as possible in habitual occlusion with the maxillary and mandibular teeth in maximum contact, and to maintain the same level of contraction during $5 \mathrm{~s}$ of recording. For each patient, the best (those with the most constant EMG signal) result is chosen, and then EMG software automatically selects $3 \mathrm{~s}$ of the MVC test, and the EMG potential is normalized as detailed before (EMG amplitude on occlusal surface divides for the mean EMG amplitude of the normalization record on the cotton rolls).

\section{Chewing test}

Chewing test is performed with chewing gum. The EMG potentials produced on each side in the first $15 \mathrm{~s}$ are recorded. Masticatory frequency and confidence ellipse of the simulta- neous differential left-right muscles activity is calculated for each movement from the potentials recorded from 4 tested muscles.

The differential left-right masseter activity represents the $x$-coordinate, and the differential temporal activity - the $y$ coordinate. From the pairs of coordinates, Hotelling's 95\% confidence ellipses (the population mean values) are calculated. In normal neuromuscular pattern, the centers of the ellipses should be located in the first and third quadrants of a Cartesian coordinate system with the same amplitude (distance of the centers of the ellipses from the origin of axes), and a $180^{\circ}$ difference between the phases. Symmetrical mastication index, masticatory frequency and impact potential of examined muscles are calculated for each patient.

\section{Neck muscle examination}

Neck muscle examination in addition to standardization recording (clenching on cotton rolls) involved standardization recording of potentials from sternocleidomastoideus muscles at maximum functional load (right-left head turns). EMG potential amplitude from sternocleidomastoideus muscles is set as $100 \%$, and relative amplitude of EMG activity of sternocleidomastoideus muscle stabilizing function at clenching is calculated.

After examination is completed we evaluated the following values:

- POC.TA - bilateral symmetry coefficient for temporalis muscles.

- POC.MM - bilateral symmetry coefficient for masseter muscles.

- TORS - lateral mandible displacement index.

- POC.SCM - bilateral symmetry coefficient for sternocleidomastoideus muscles.

- CER.LOAD - sternocleidomastoideus muscle functional activity coefficient.

- IMPACT - impact potential of examined muscles.

POC. The EMG potentials from the paired muscles are compared by calculating a percentage overlapping coefficient (POC, \%) [10]. POC is an index of the symmetric distribution of the muscular activity as determined by occlusion; it is computed by superimposing the left and right side normalized EMG amplitudes of a muscle (masseter or temporalis) over time: the area of superimposition is assessed as a percentage of the total EMG amplitudes. The index ranges between $0 \%$ and $100 \%$ : when two paired muscles contract with perfect symmetry, POC is $100 \%$ (EMG amplitudes completely superimposed). Mean (masseter and temporalis) POCs are obtained for each patient. POC over $80 \%$ is correlated to symmetrical muscle function.

TORS. Contralateral masseter and temporalis muscles (for instance, right temporalis and left masseter) form a muscular couple [10]. If only one muscular couple is activated, unbalanced by a similar contraction of the other muscular couple, a potential lateral displacement of the mandible might occur. Torque coefficient (TORS, \%) [10] is calculated by superimposing the right temporalis plus left masseter normalized EMG amplitudes over the left temporalis plus right masseter 
normalized EMG amplitudes; the area of superimposition is assessed as a percentage of the total EMG amplitudes. TORS ranges between $0 \%$ (complete presence of lateral displacing force) and $100 \%$ (no lateral displacing force). Under normal circumstances, TORS value is below $10 \%$.

CER.LOAD. Is assessed as the percentage ratio between the SCM muscle potentials recorded during MVC (stabilization function) and the muscle potentials obtained during the maximum contraction standardization task (head turns). Normally, CER.LOAD value is below $20 \%$.

IMPACT. Absolute values vary between 500 and $2500 \mathrm{mV}$.

$A T T I V$. Is defined a ratio of total bilateral temporalis muscle biopotential at MVC to total bilateral masseter muscles biopotential. ATTIV values range from 0 to $10 \%$ in physiological occlusal situations. Negative ATTIVE values in patients with malocclusion might reflect participation of frontal teeth in mastication and lack of lateral occlusion support.

\section{Case report}

A female patient, 23 years old, presented with complaints of fatigue and paining masseter muscles. The symptoms deteriorated after food intake. In addition, the patient reported discomfort while clenching, sleeping disorders and headaches. Orthodontic treatment was completed shortly before. A consultation with a specialist was recommended to her because of the symptoms. The situation at the first visit is shown in Figs. 1-3.

Clinical functional analysis was performed with the following results: mouth opening unrestricted; left masseter muscle tender and spastic; pain on palpation in the region of the left maxillary tuber. Owing to pain at clenching and inability to determine a stable intercuspal position (ICP), the clinical evaluation of static occlusion was demanding and the results of these tests were not reproducible. Assessment of dynamic occlusion showed canine dominance on both sides and sufficient protrusive control. Lateral cephalogram showed dentoalveolar compensation in the lower anterior dental arch. The patient was preliminary diagnosed with myofascial face pain syndrome after complete clinical examination.

4-channel EMG showed temporalis muscles asymmetry (POC $77.7 \%$, normal value $>80 \%$ ) with left temporalis predominance and left lateral displacement (TORS 13.5\%, normal value $<10 \%$ ). ATTIV value, relevant for muscular cofunction in sagittal plane, was $-34.6 \%$ (Fig. 4). These findings indicated dominance of temporalis muscles at clenching. ATTIV values range from 0 to $10 \%$ in patients with Angle class I occlusion, indicating masseter muscles predominance. Impact potential reached $1446 \mathrm{mV}$ and was consistent with population mean normal values. Analysis of mastication protocol showed mastication symmetry of $2.05 \%$, masticatory frequency of 0.93 cycle per second on the left side and 1.0 cycle per second on the right side. Masticatory pattern on the left utilized $75.1 \%$ of ipsilateral muscular activity and $24.9 \%$ of balancing activity (normal values $60-70 \%$ on the working side and $40-30 \%$ on the balancing side). Masticatory pattern on the right utilized 41.7 and $58.3 \%$ of activities respectively.
Three quarters of the Hottelning's 95\% confidence ellipses corresponded to the left temporalis and masseter muscles. Thus, mastication on both sides was characterized by the leftside muscular predominance. Impact potentials during mastication reached $1212.2 \mathrm{mV}$ on the right side and $543.5 \mathrm{mV}$ and the left sides. The mean masticatory amplitude on the

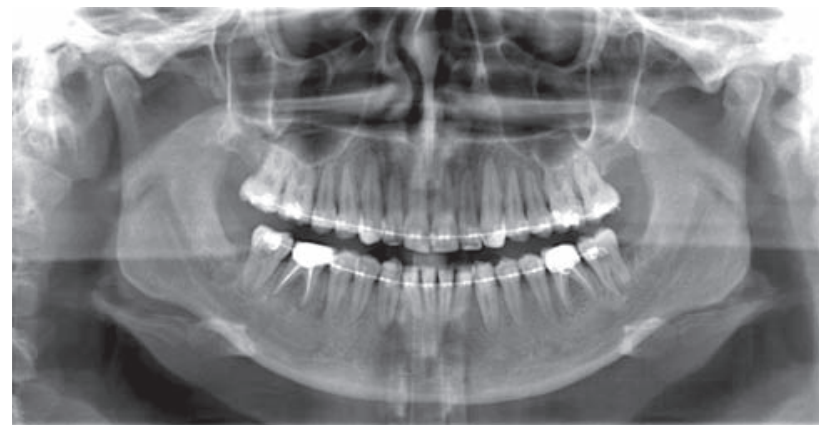

Fig. 1: The initial panoramic x-ray. A 23-year-old female patient presented after orthodontic treatment with signs and symptom of myofascial pain syndrome. For details see text

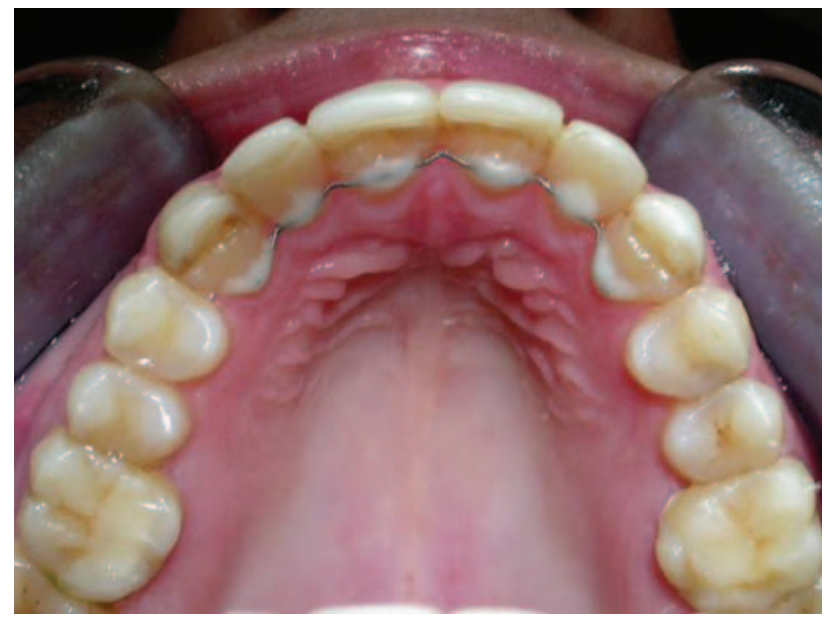

Fig. 2: Occlusal view of upper jaw. For details see text

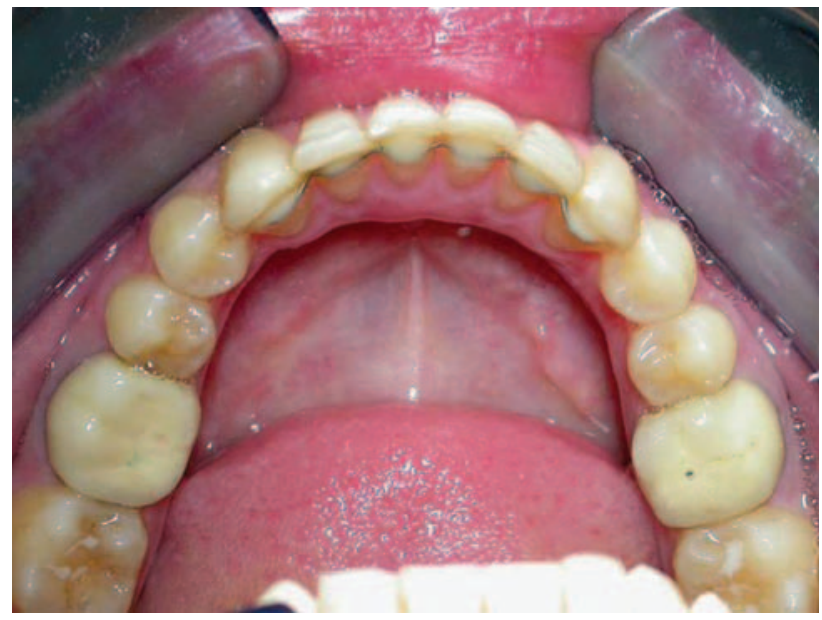

Fig. 3: Occlusal view of lower jaw. For details see text 
POC - Percent Overlapping Coefficient POCtemp: $77.67 \%$ (Sx) POCmass: $85.16 \%$ (Sx)

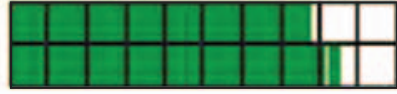

POCmedio: $81.42 \%$

\begin{abstract}
ASIM: $\quad-17.30 \%$
ATTIV: $\quad-34.61 \%$
\end{abstract}

TORQUE: $-11.21 \%$

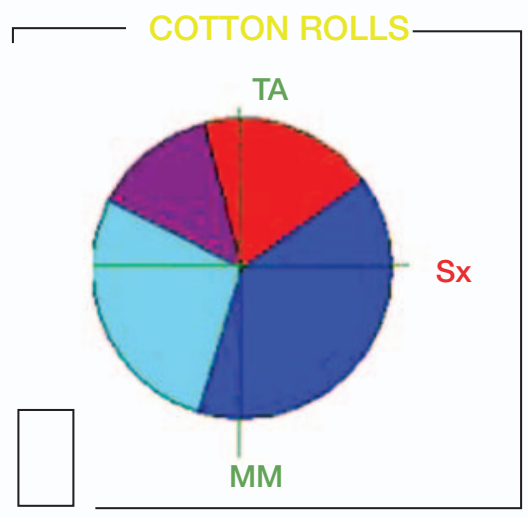

TA: $\quad 59.8 \mu \mathrm{V}$

$73.9 \mu \mathrm{V}$

MM: $126.1 \mu \mathrm{V}$

$145.6 \mu \mathrm{V}$

IMP:

$1217 \mu \mathrm{V}^{*} \mathrm{sec}$

Acquisizione (data): 02.06 .2007

Code:

0004

Patient:

Галина Черных

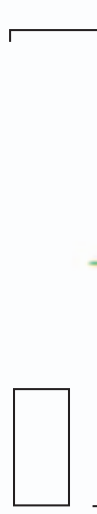

$84.2 \mu \mathrm{V}$

$89.6 \mu \mathrm{V}$

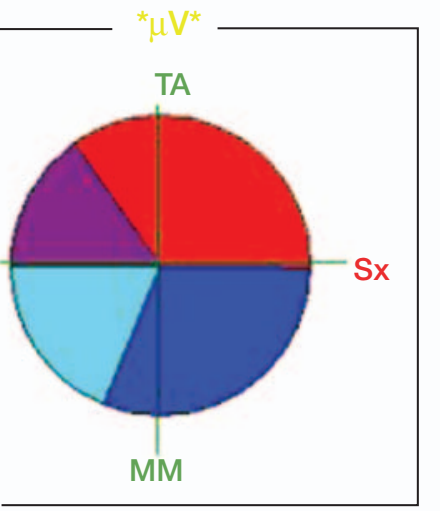

$160.0 \mu \mathrm{V}$

$138.2 \mu \mathrm{V} \quad 78.0 \%$

$1446 \mu \mathrm{V}^{*} \mathrm{sec}$

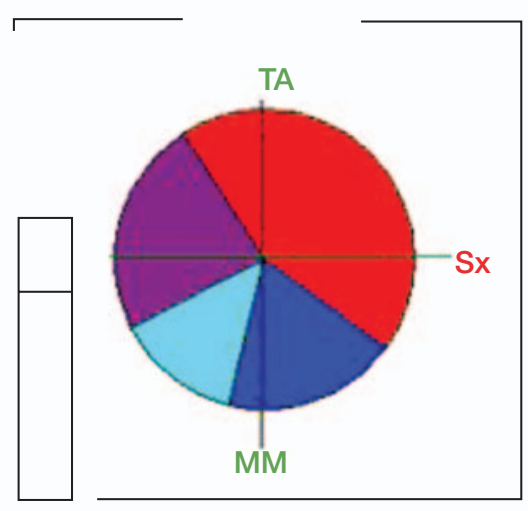

$216.7 \%$

$94.9 \%$

Time sampling: $25 \mathrm{~ms}$

Periodo: $\quad 3000 \mathrm{~ms}$

File: 0004.2.cle

Fig. 4: Results of the initial EMG examination. The Percentage Overlapping Coefficient (POC) is shown. 4-channel EMG showed temporalis muscles asymmetry (POC $77.7 \%$, normal value $>80 \%$ ) with left temporalis predominance and left lateral displacement (TORS $13.5 \%$, normal value $<10 \%$ ). ATTIV value, relevant for muscular co-function in sagittal plane, was $-34.6 \%$ (Fig. 4). These findings indicated dominance of temporalis muscles at clenching. For details see text

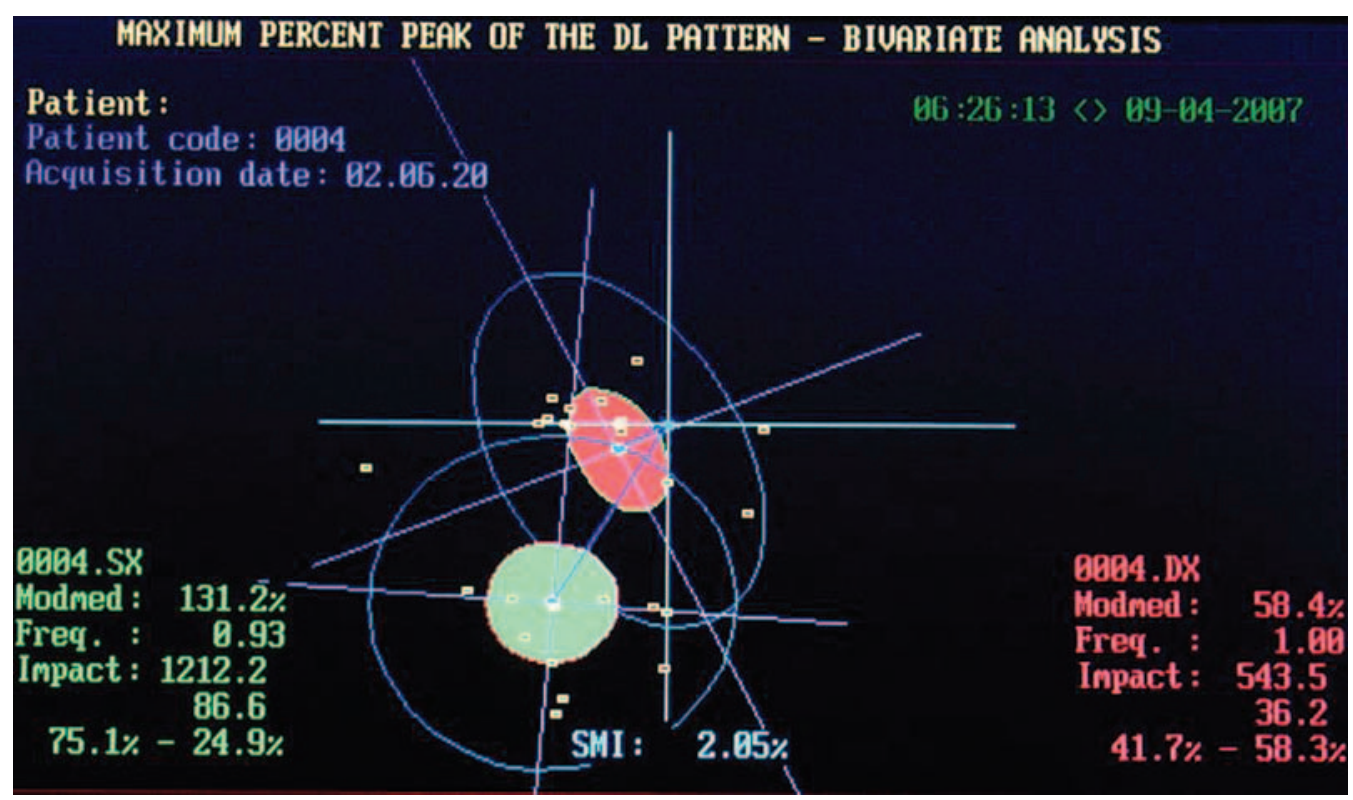

Fig. 5: Results of the initial EMG examination. Masticatory pattern on the right utilized 41.7 and $58.3 \%$ of activities respectively. Three quarters of the Hottelning's $95 \%$ confidence ellipses corresponded to the left temporalis and masseter muscles. Impact potentials during mastication reached $1212.2 \mathrm{mV}$ on the right side and $543.5 \mathrm{mV}$ on the left side. The mean masticatory amplitude on the right side is $131.2 \%$ and $58.4 \%$ on the left side. The absolute values in addition proved predominance of mean masseter and temporal muscular electrical activity on the left side (86.6 mV and $36.2 \mathrm{mV}$ in each masticatory cycle. For details see text 
right side was approximately 2 -fold increased in comparison to the left side (131.2 and $58.4 \%$ respectively) and absolute values in addition proved predominance of mean masseter and temporal muscular electrical activity on the left side ( $86.6 \mathrm{mV}$ and $36.2 \mathrm{mV}$ in each masticatory cycle on the left

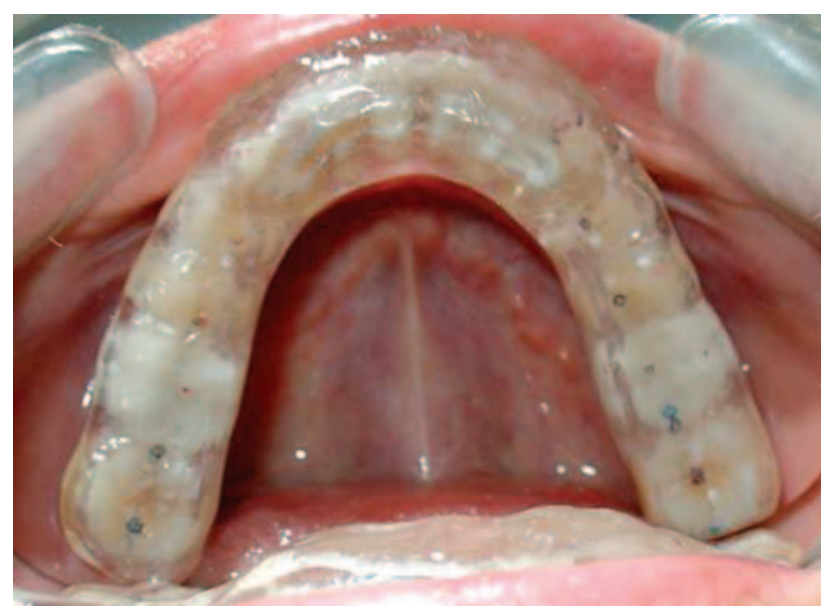

Fig. 6: Occlusal view of the splint used in the initial phase of the treatment. This phase lasted 14 days with subsequent equilibration of the splint. For details see text and on the right respectively) (Fig. 5). EMG data were consistent with clinical signs and symptoms of stage I myofascial face pain syndrome.

Upper jaw model was mounted into Protar-KAVO ${ }^{\circledR}$ system. Mechanic axiography was performed with ARCUS-Pro-KAVO ${ }^{\circledR}$. Mandibular model transfer was performed with wax bite registration in reference position. Functional analysis in the articulator demonstrated static contacts at teeth $35,44,45$ and 47 with all the other teeth not in contact and impairment of static occlusion. A causal relation to myofascial pain syndrome was concluded and a muscle relaxation mandibular splint was prescribed (Fig. 6). The pain improved significantly within 14 days of splint therapy. 4-channel EMG showed normal temporal and masseter muscle symmetry. A displacement of the mandible was not found anymore. ATTIV and IMPACT values stayed within normal limits. Analysis of mastication protocol showed mastication symmetry of $17.78 \%$, masticatory frequency of 1.07 cycles per second on the left side and 0.93 cycles per second on the right side. Masticatory pattern on the left utilized $84.9 \%$ of ipsilateral muscular activity and $15.1 \%$ of balancing activity. Masticatory pattern on the right utilized $54.3 \%$ and $45.7 \%$ of activities respectively (initial values: 41.7 and 58.3\% respectively). Hottelning's 95\% confidence ellipses corresponded to the left and right masseter muscles; however, both ellipses were located in

POC - Percent Overlapping Coefficient

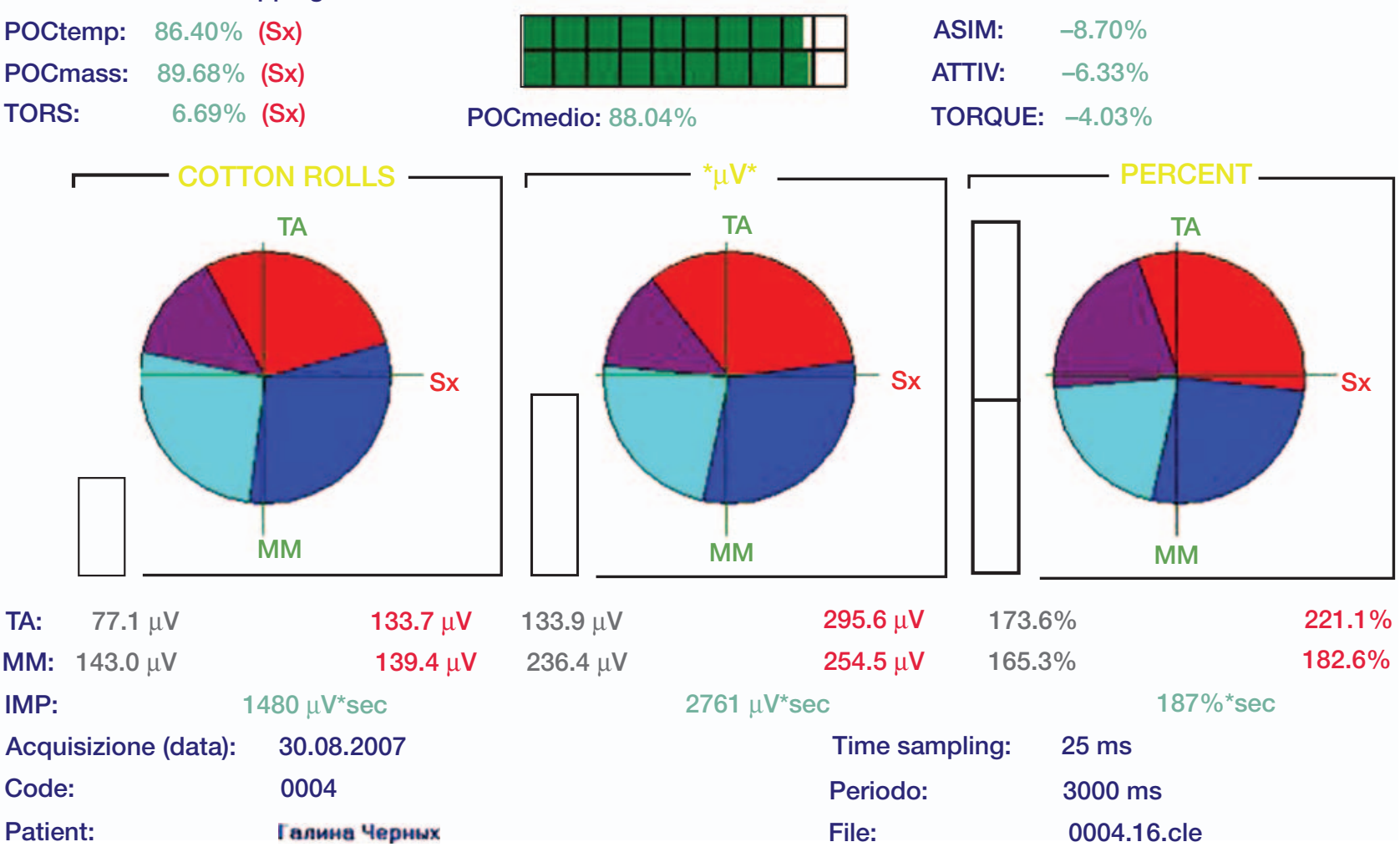

Fig. 7: Results of the control EMG examination after the restorative treatment. ATTIV and IMPACT values stayed within normal limits. Analysis of mastication protocol showed mastication symmetry of $17.78 \%$, masticatory frequency of 1.07 cycles per second on the left side and 0.93 cycles per second on the right side. Masticatory pattern on the left utilized $84.9 \%$ of ipsilateral muscular activity and $15.1 \%$ of balancing activity. Masticatory pattern on the right utilized $54.3 \%$ and $45.7 \%$ of activities respectively. A remarkable change can be observed by comparing Fig. 7 with Fig. 4 . For details see text 


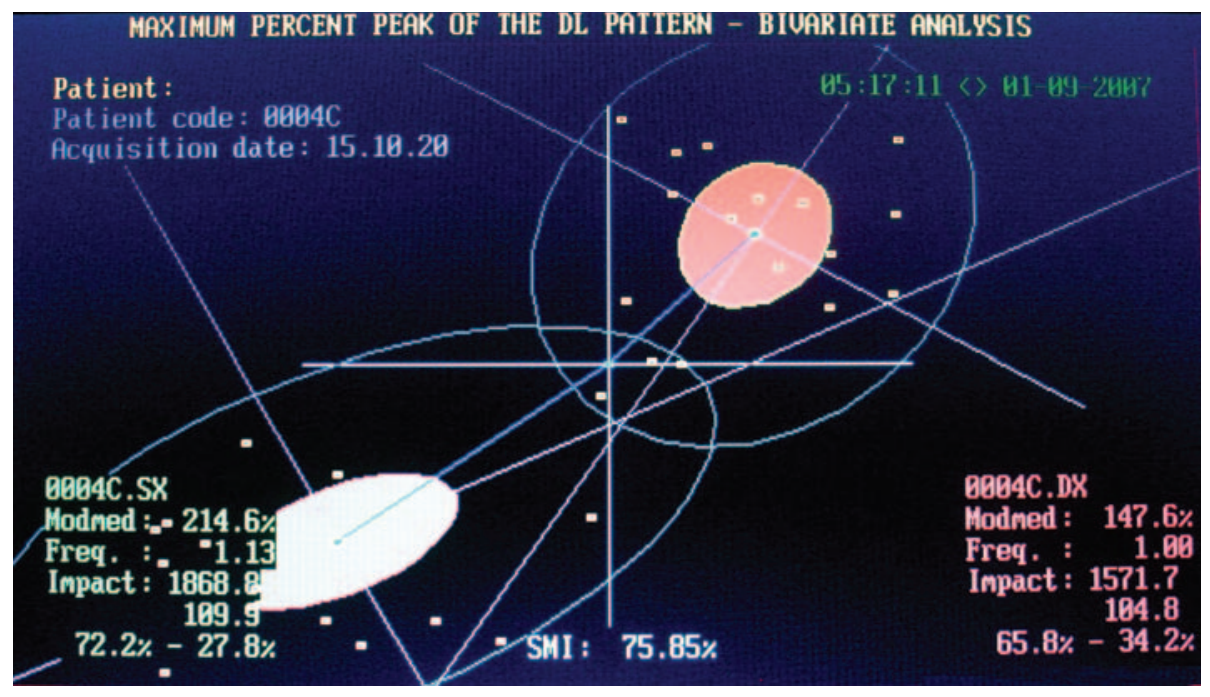

Fig. 8: Results of the control EMG examination after the restorative treatment. Hottelning's 95\% confidence ellipses corresponded to the left and right masseter muscles; however, both ellipses were located in the zone of the left temporalis muscle. Impact potentials during mastication reached $1062.3 \mathrm{mV}$ and $884.9 \mathrm{mV}$ on the right and the left side respectively. Mean masticatory amplitude on the right side was $66.4 \mathrm{mV}$ and $57.5 \mathrm{mV}$ in each masticatory cycle on the left and on the right respectively. A remarkable change can be observed by comparing Fig. 8 (after treatment) with Fig. 5 (before treatment). For details see text

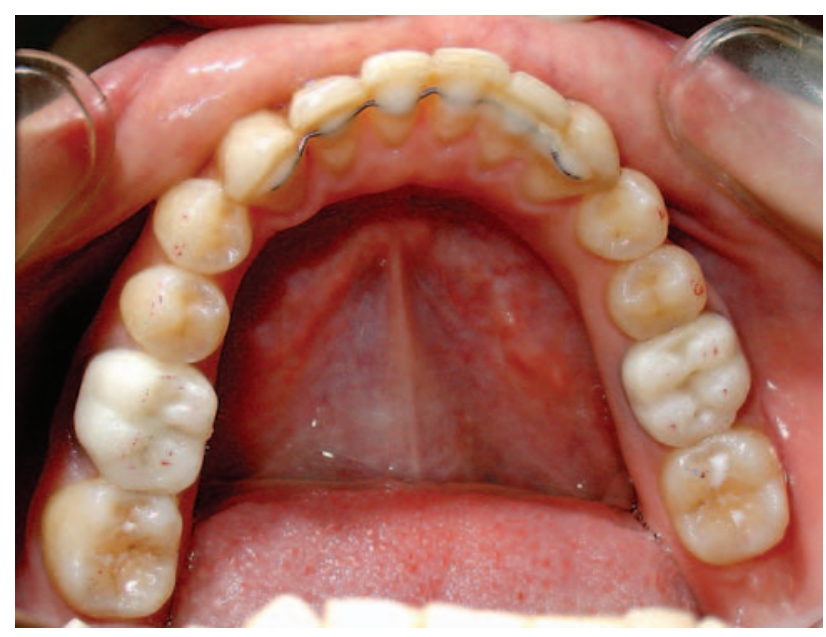

Fig. 9: Occlusal view after the restoration. Sufficient and adequate centric stops have been established. For details see text

the zone of the left temporalis muscle. Impact potentials during mastication reached $1062.3 \mathrm{mV}$ and $884.9 \mathrm{mV}$ on the right and the left side respectively (initial values: $1212.2 \mathrm{mV}$ and $543.5 \mathrm{mV}$ respectively). Mean masticatory amplitude on the right side was $66.4 \mathrm{mV}$ and $57.5 \mathrm{mV}$ in each masticatory cycle on the left and on the right respectively (Figs. 7, 8).

Thus, mastication symmetry was restored by activation of the right masseter while the right temporalis muscle did not take any part in mastication (Fig. 14). At this stage, treatment phase 2 was started. Patient was recommended to wear the splint only at night to stabilize occlusion. The restorative treatment was completed within 2 months. Adequate centric contacts in the buccal sections on both sides of the jaw were observed at ICP. Manbibular first molars were not in occlusal contact at this moment because of endodontic retreatment
(Fig. 9). Two months later, the patient did not report any complaints nor presented with whichever clinical signs and symptoms of impaired function. 4-channel EMG demonstrated all values within normal range, except for impact potential that reached $2767 \mathrm{mV}$. We interpreted this finding with a tendency to bruxism. Analysis of mastication protocol showed mastication symmetry of $75.85 \%$, masticatory frequency of 1.13 cycles per second on the left side and 1.0 cycle per second on the right side with good amplitudes and phases. Masticatory pattern on both sites was utilized predominantly by temporalis muscles that were regarded as normal model of neuromuscular compensation consistent with class II malocclusion. Impact potentials during mastication reached $1868 \mathrm{mV}$ and $1571 \mathrm{mV}$ on the right and the left side respectively.

\section{Conclusion}

The application of EMG in clinical practice is useful in the following situations:

1. Screening for functional activity of chewing muscles disorders.

2. Development of evidence-based treatment protocols.

- to control static and dynamic occlusion in diagnostics,

- to control static and dynamic occlusion during reconstructive procedures,

- to control neuromuscular balance before and after orthodontic treatment.

3. Diagnosis of combined dental and neurological pathology. 4. Medical expertise.

\section{Conflict of interest}

The authors declare that there is no conflict of interest. 


\section{References}

[1] Zenkov LR, Ronkin MA. Functional diagnostics of diseases of nervous system. Moscow: Moscow Medicine; 1991. p. 623.

[2] Rumyantseva MF, Loseva TN, Bunina TP. Practical training manual on physiology including basis of anthroponomy. Moscow: Moscow Medicine; 1986.

[3] Khvatova VA. Functional diagnostics and therapy in dentistry. Moscow: Medical Book; 2007. pp. 73-85.

[4] Khvatova VA. Clinical gnathology. Moscow: Moscow Medicine; 2005. pp. 114-7.

[5] Blanksma NG, van Eijden TM. Electromyographic heterogeneity in the human temporalis and masseter muscles during static biting, open/close excursions, and chewing. J Dent Res 1995; 74:1318-27.

[6] Ferrario V, Sforza C. Biomechanical model of the human mandible: a hypothesis involving stabilizing activity of the superior belly of lateral pterygoid muscle. J Prosthet Dent 1992;68(5):829-33.

[7] Ferrario VF, Sforza C, Miani AJR, Serrao G. Kinesiographic 3-dimensional evaluation of mandibular border movements: a statistical study in a normal young non-patient group. J Prosthet Dent 1992;68:672.

[8] Ferrario VF, Sforza C, Colombo A, Ciusa V. An electromyographic investigation of masticatory muscles symmetry in normo-occlusion subjects. J Oral Rehabil 2000;27:33.

[9] Ferrario VF, Sforza C. Biomechanical model of the human mandible in unilateral clench: distribution of TMJ reaction forces between working and balancing sides. J Prosthet Dent 1994;72:169.

[10] Ferrario VF, Sforza C, Tartaglia GM, Colombo A, Serrao G. Size and shape of the human first permanent molar: a Fourier analysis of the occlusal and equatorial outlines. Am J Phys Anthropol 1999;108:281. 